\title{
Efficacy and safety of belimumab in the treatment of systemic lupus erythematosus: A meta-analysis
}

\author{
JIACHEN ZHANG ${ }^{1 *}$, XIANGYING LIU ${ }^{2 *}$, YAN XU ${ }^{3}$, HONGYAN $\mathrm{LI}^{3}$ and YUEXIA XU ${ }^{3}$ \\ ${ }^{1}$ Department of Nephrology, Taizhou Hospital of Zhejiang Province Affiliated to Wenzhou Medical University, \\ Taizhou, Zhejiang 317000; ${ }^{2}$ Department of Nephrology, Changzhou Geriatric Hospital Affiliated to Soochow University, \\ Changzhou No. 7 People's Hospital, Changzhou, Jiangsu 213000; ${ }^{3}$ Department of Nephrology, \\ Suzhou Ninth Hospital Affiliated to Soochow University, Suzhou, Jiangsu 215200, P.R. China
}

Received October 4, 2021; Accepted February 1, 2022

DOI: 10.3892/wasj.2022.143

\begin{abstract}
The present meta-analysis aimed to systematically review the efficacy and safety of belimumab (BLM) therapy for patients with active and autoantibody-positive systemic lupus erythematosus (SLE) treated with standard of care (SOC). To evaluate the efficacy and safety of BLM plus SOC treatment in patients with active SLE, eligible studies were retrieved from the Web of Science, The Cochrane Library, PubMed and Embase online databases up to July, 2021. Review Manager (version 5.3) and STATA 16.0 software were used to analyze the extracted data from the included studies. A total of seven randomized controlled trials (RCTs) and 19 case series, with 4,235 and 2,597 patients with SLE, respectively were analyzed. In the RCTs, there were more SLE responder index (SRI-4) responders in the BLM group compared with the control group [52.8 vs. $41.6 \%$; relative risk (RR), 1.27 ; $95 \%$ confidence interval (95\% CI), 1.18-1.37); $\mathrm{P}<0.00001]$. In addition, compared with the placebo group, more patients in the BLM group achieved a $\geq 4$-point reduction in the Safety of Estrogens in Lupus National Assessment-Systemic Lupus Erythematosus Disease Activity Index score (RR, 1.26; 95\% CI, 1.15-1.38; P<0.00001). Furthermore, treatment with BLM was found to significantly decrease the risk of severe disease exacerbations (flares) compared with the control group (RR, 0.72; 95\% CI, 0.63-0.81; $\mathrm{P}<0.00001$ ). Additionally, the corticosteroid dosage was reduced by $\geq 25$ or $50 \%$ to $\leq 7.5 \mathrm{mg} /$ day during weeks $40-52$ in more patients in the BLM group compared with the control group (RR,
\end{abstract}

Correspondence to: Dr Xiangying Liu, Department of Nephrology, Changzhou Geriatric Hospital Affiliated to Soochow University, Changzhou No. 7 People's Hospital, 288-7 Yanling East Road, Changzhou, Jiangsu 213000, P.R. China

E-mail: 18896778783@163.com

*Contributed equally

Key words: systemic lupus erythematosus, belimumab, meta-analysis, efficacy, safety
1.44; 95\% CI, 1.17-1.76; P=0.0005). However, no differences were observed in the RR of adverse events (AEs) and severe AEs between both groups (RR, 1.00; 95\% CI, 0.97-1.02; $\mathrm{P}=0.84$; and $\mathrm{RR}, 0.80 ; 95 \% \mathrm{CI}, 0.62-1.03 ; \mathrm{P}=0.08$, respectively). In the case series studies, the total remission rate was $60.5 \%$ (95\% CI, 52.1-68.3\%; P=0.015). In addition, treatment with BLM significantly decreased the use of corticosteroids (mean deviation, -8.73 ; 95\% CI, -11.07 to -6.39 ; $\mathrm{P}<0.00001)$. Overall, the results of the present meta-analysis demonstrated that BLM therapy provided significant clinical efficacy and it was well-tolerated by patients with active SLE. More importantly, treatment with BLM may reduce the use of glucocorticoids.

\section{Introduction}

Systemic lupus erythematosus (SLE) is a complex systemic autoimmune disease that predominantly affects women and is characterized by clinical heterogeneity, an unpredictable course and disease exacerbations (flares). The aim of SLE therapy is to achieve disease remission, or at least a low disease activity and a prevention of flares. Therefore, the treatment approaches for SLE mainly include high-intensity immunosuppressive therapy for an initial period followed by a less intensive treatment strategy to prevent relapses for a long period of time. Drug options include cyclophosphamide, mycophenolate mofetil, azathioprine and calcineurin inhibitors, in combination with glucocorticoids. For patients with refractory lupus or life-threatening disease, biologics, combination regimens, plasma exchange and intravenous immunoglobulins are adopted (1). Due to persistent disease activity or flares, a large proportion of patients with SLE require long-term treatment with corticosteroids and/or immunosuppressive drugs, eventually leading to the progressive aggravation of the impairment and adverse outcomes. However, these therapies are often not sufficiently effective.

B lymphocyte stimulator (BLyS), also known as B-cell activating factor (BAFF), is a member of the TNF family and plays a significant role in B-cell survival. It has been reported that BLyS expression is upregulated in patients with autoimmune diseases, such as SLE $(2,3)$. Belimumab (BLM), a human IgG monoclonal antibody, binds with soluble human BLyS to 
inhibit its biological activity, thereby promoting autoimmune $\mathrm{B}$ cell apoptosis and reducing the number of new or existing autoimmune B-cell clones $(4,5)$.

An increasing number of clinical trials have supported the beneficial effects of BLM in the treatment of SLE. Therefore, BLM combined with standard of care (SOC) treatment was approved by the Food and Drug Administration (FDA) in 2011 for the treatment of patients with active and autoantibody-positive SLE (6). In addition, BLM has recently been approved for the treatment of children $>5$ years of age suffering from childhood-onset SLE (cSLE). However, the therapeutic effects and adverse reactions of the aforementioned drug vary with the extension of the treatment duration. Nevertheless, comprehensive meta-analyses on the efficacy and safety of BLM in patients with active SLE remain limited (7-9). Therefore, the present meta-analysis aimed to systematically review and summarize these studies to evaluate the efficacy and safety of the use of BLM in patients with active SLE treated with SOC.

\section{Data and methods}

Sources and searches. Electronic literature screening was performed using the Web of Science, The Cochrane Library, PubMed and Embase databases with a cut-off date of July, 2021. The Medical Subject Headings terms used were as follows: 'lupus', 'systemic lupus erythematosus', 'SLE', 'belimumab' and 'Benlysta'. Only studies published in English were included. In the case of overlapping studies from the same authors, the most recent or complete study was included in the meta-analysis.

Inclusion and exclusion criteria. The inclusion criteria were as follows: i) Patients diagnosed with SLE according to the American College of Rheumatology classification criteria (10); ii) randomized controlled trials (RCTs) or case series studies, including prospective and retrospective case series; iii) patients with SLE treated with BLM; iv) patients positive for SLE autoantibodies (antinuclear and/or anti-dsDNA autoantibodies); v) patients treated with a stable treatment regimen prior to the initiation of the trial, including corticosteroids, antimalarials, immunosuppressive and non-steroidal anti-inflammatory drugs; vi) patients with active SLE, defined by a Safety of Estrogens in Lupus National Assessment-Systemic Lupus Erythematosus Disease Activity Index (SELENA-SLEDAI) score of $\geq 8$ or 6 . The exclusion criteria were as follows: i) Case report studies and review articles; ii) studies with no available data; iii) studies that included patients who were previously treated with B-lymphocyte-targeting drugs or other novel medications against SLE, other than corticosteroids.

Data extraction and quality assessment. Two investigators, XL and JZ independently screened the titles and abstracts of the included studies, identified the duplicates studies, reviewed the full articles, decided on the eligibility of the studies and collected the data. Disagreements were resolved through discussion. XL designed a standard electronic format for data collection. The following data were extracted from the eligible trials: Study design, sample size, age, female proportion, disease duration, use of other immunosuppressive agents, medication protocols, outcome characteristics, adverse events (AEs) and severe AEs (SAEs). The quality of each trial was evaluated according to the Cochrane Collaboration tool (https://training.cochrane. org/handbook/current).

Statistical analyses. STATA 16.0 (Stata Corp LP) and Review Manager (version 5.3; The Nordic Cochrane Centre) software were used to pool and analyze the results, respectively. More specifically, the response rate of the case series studies was pooled using STATA 16.0 (Stata Corp LP), while additional data analysis was performed using Review Manager (version 5.3; The Nordic Cochrane Centre). For binary outcomes, the relative risk (RR) was estimated with a $95 \%$ confidence interval (95\% CI). The results are presented as analytical graphs generated using forest plots. Heterogeneity was assessed using a $\chi^{2}$-based Q test. Therefore, with an $\mathrm{I}^{2}<50 \%$ or $\mathrm{P}>0.10$, heterogeneity was considered small and RR values were pooled in a fixed-effects model. With an $\mathrm{I}^{2}>50 \%$ or $\mathrm{P}<0.10$, heterogeneity was considered significant and RR values were pooled in a random effects model. A value of $\mathrm{P}<0.05$ was considered to indicate a statistically significant difference.

\section{Results}

Search results. The flow diagram of trial selection is presented in Fig. 1. Among a total of 2,377 eligible manuscripts, after screening, seven RCTs and 19 case series were included in the meta-analysis (11-36).

Characteristics of included studies. A total of seven RCTs (11-17) and 19 case series (18-36) were included in the analysis, including 6,832 patients with SLE. The details of the aforementioned studies are presented in Tables I and II. As regards the RCTs, six trials $(11-15,17)$ included adult patients only, and one (16) trial only children. In addition, in six trials (11-14,16,17), BLM was administered intravenously and in one trial subcutaneously (15).

Quality evaluation of the literature. All eligible RCTs included multicenter, randomized, double-blind and placebo-controlled studies. Among the eligible trials, four studies $(12-14,16)$ described the random method, while the other three $(11,15,17)$ did not mention a specific method. A quality evaluation of the included studies is presented in Table III.

$S L E$ responder index (SRI) rate in RCTs. In six RCTs (12-17), a SRI-4 response rate was reported at the end of each study. There was homogeneity among these studies $\left(\mathrm{I}^{2}=0 \% ; \mathrm{P}=0.91\right)$. Compared with the control group, treatment with BLM resulted in a significantly increased SRI-4 response rate [52.8\% (1,306/2,474) vs. $41.6 \%(518 / 1,246)$; RR, 1.27; 95\% CI, 1.18-1.37; $\mathrm{P}<0.00001 ;$ Fig. 2].

SELENA-SLEDAI score $\geq 4$-point reduction in RCTs. In four RCTs $(12-14,16)$, the SELENA-SLEDAI score was reduced by at least 4 points in patients with SLE. In addition, there was homogeneity among these studies $\left(\mathrm{I}^{2}=0 \% ; \mathrm{P}=0.91\right)$. The number of patients who achieved at least a 4-point reduction in the SELENA-SLEDAI score was significantly 

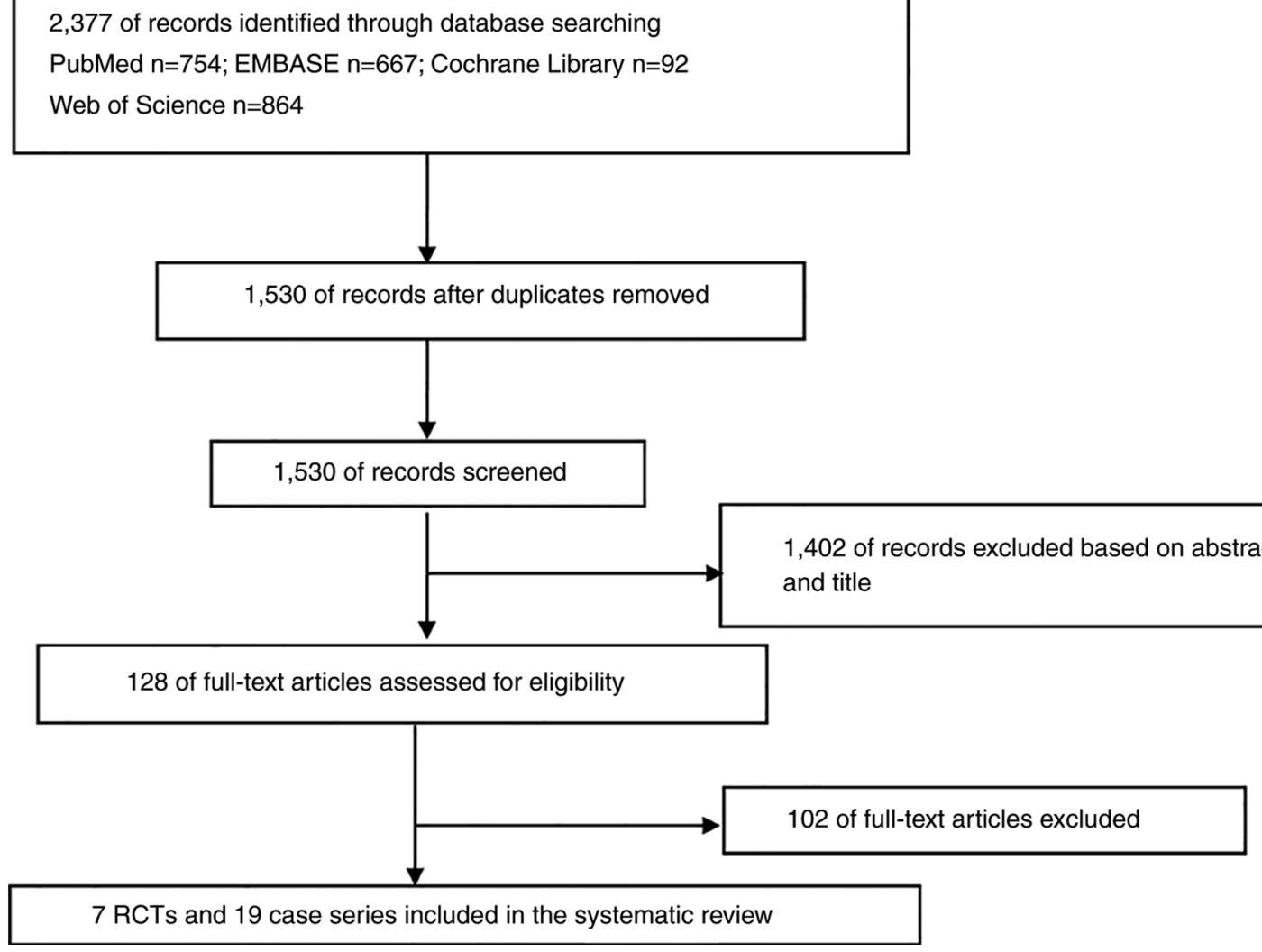

Figure 1. Flow diagram of trial selection used in the present meta-analysis.

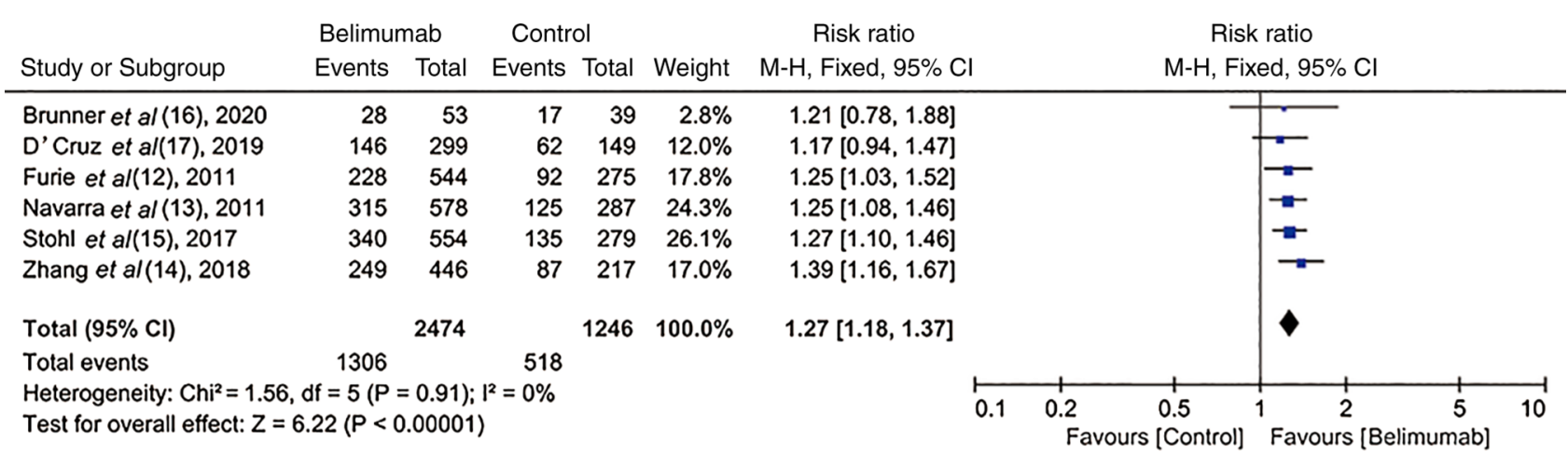

Figure 2. Meta-analysis results of systemic lupus erythematosus responder index 4 response between the belimumab and control groups.

increased in the BLM group compared with the control group $[52.0 \%(843 / 1,622)$ vs. $41.3 \%(338 / 819) ; \mathrm{RR}, 1.26 ; 95 \% \mathrm{CI}$, 1.15-1.38); $\mathrm{P}<0.00001$; Fig. 3].

Change in severe flares in RCTs. Based on the modified SLE flare index (37-39), severe flares were reported in all trials (11-17). However, the heterogeneity among these studies was poor $\left(\mathrm{I}^{2}=44 \% ; \mathrm{P}=0.10\right)$. Patients treated with $\mathrm{BLM}$ exhibited a lower rate of severe flares compared with the placebo group [17.2\% (485/2,817) vs. 23.4\% (320/1,370); RR, 0.72; 95\% CI, 0.63-0.81); P<0.00001; Fig. 4].

Corticosteroid dosage reduction in RCTs. In six RCTs $(11-15,17)$, the average dose of corticosteroids was reduced by $\geq 25$ or $50 \%$ to $\leq 7.5 \mathrm{mg}$ /day during weeks $40-52$ in the majority of patients treated with BLM. There was significant homogeneity among these studies $\left(\mathrm{I}^{2}=0 \% ; \mathrm{P}=0.93\right)$. More specifically, the majority of patients in the BLM group $[18.6 \%(305 / 1,642)]$ were treated with a reduced dose of corticosteroids (reduced by $\geq 25$ or $50 \%$ to $\leq 7.5 \mathrm{mg}$ /day) at weeks $40-52$ compared with those in the control group [12.8\% (104/813); RR, 1.44; 95\% CI, 1.17-1.76); $\mathrm{P}=0.0005$; Fig. 5].

Safety and tolerability of BLM in RCTs. All trials (11-17) recorded the AEs and SAEs. The incidence of AEs and SAEs, including arthralgia, fatigue, mortality and infection, was similar between the BLM and control groups (RR, 1.00; 95\% CI, 0.97-1.02; $\mathrm{P}=0.84$ vs. RR, 0.80; 95\% CI, 0.62-1.03; $\mathrm{P}=0.08$; Figs. 6 and 7), thus indicating that BLM was well tolerated. 


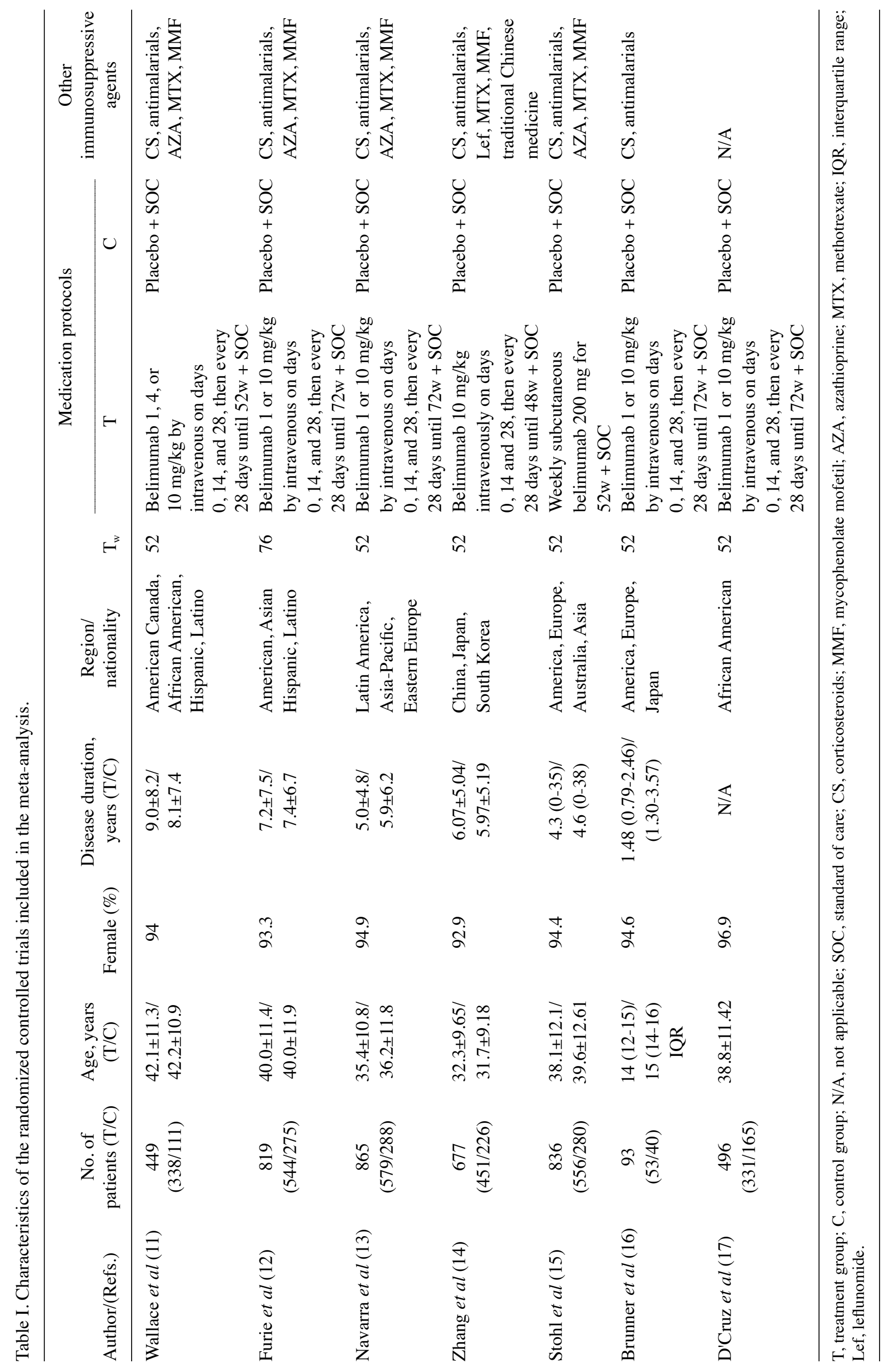




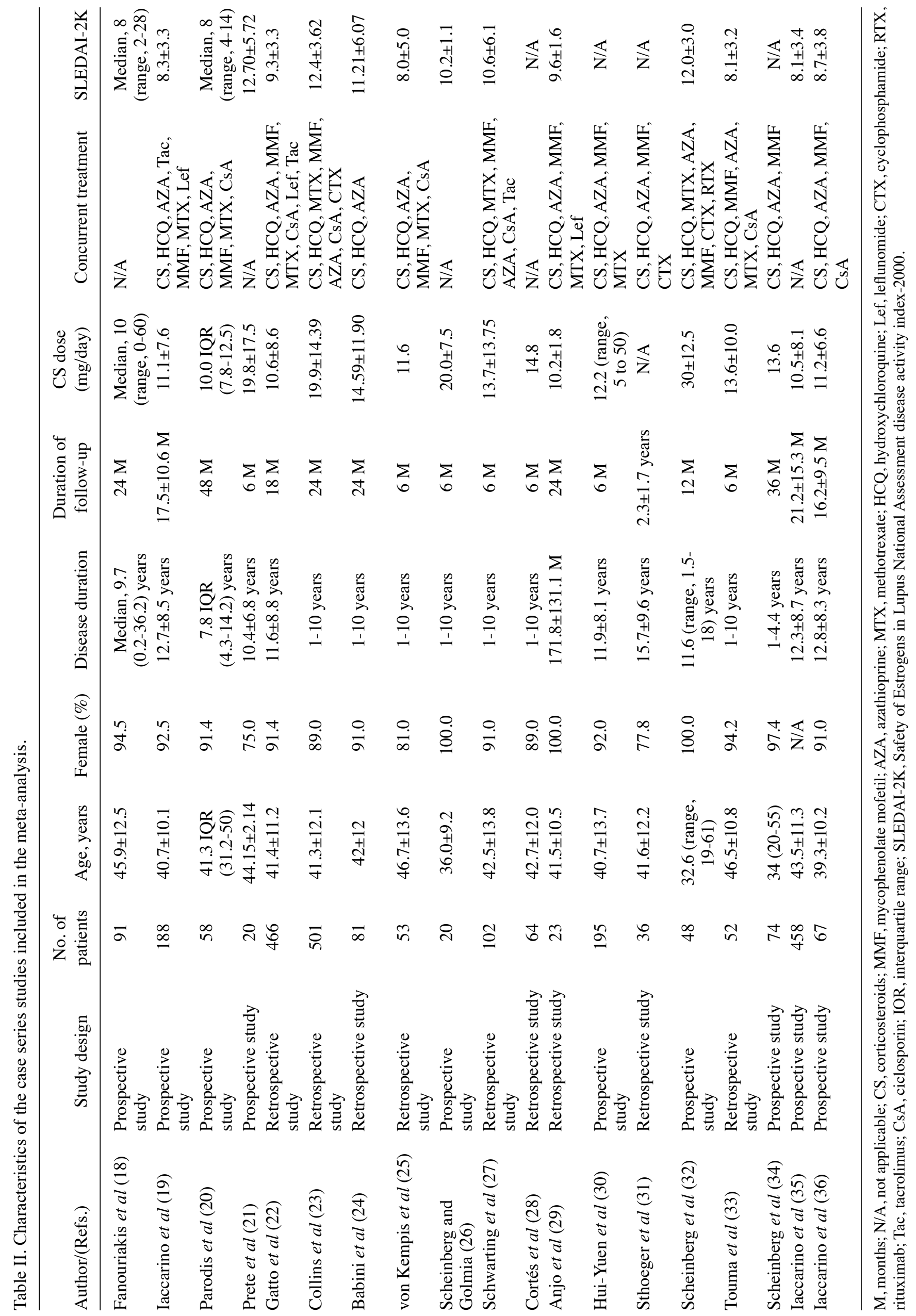


Table III. Assessment of methodological quality of included studies.

\begin{tabular}{|c|c|c|c|c|c|c|}
\hline Study & Random allocation & Blind method & $\begin{array}{c}\text { Hidden } \\
\text { distribution }\end{array}$ & $\begin{array}{l}\text { The } \\
\text { completeness of } \\
\text { the result data }\end{array}$ & $\begin{array}{l}\text { Selective } \\
\text { reporting } \\
\text { of results }\end{array}$ & Other bias \\
\hline Wallace et al (11) & Mention random & Double-blind & Not clear & Yes & No & Not clear \\
\hline Furie et al (12) & $\begin{array}{l}\text { Central interactive } \\
\text { voice response system }\end{array}$ & Double-blind & Yes & Yes & No & Not clear \\
\hline Navarra et al (13) & $\begin{array}{l}\text { Central interactive } \\
\text { voice response system }\end{array}$ & Double-blind & Yes & Yes & No & Not clear \\
\hline Zhang et al (14) & Validated software & Double-blind & Yes & Yes & No & Not clear \\
\hline Stohl et al (15) & Mention random & Double-blind & Not clear & Yes & No & Not clear \\
\hline Brunner et al (16) & $\begin{array}{l}\text { Interactive response } \\
\text { system }\end{array}$ & Double-blind & Yes & Yes & No & Not clear \\
\hline D'Cruz et al (17) & Mention random & Double-blind & Not clear & Yes & No & Not clear \\
\hline
\end{tabular}

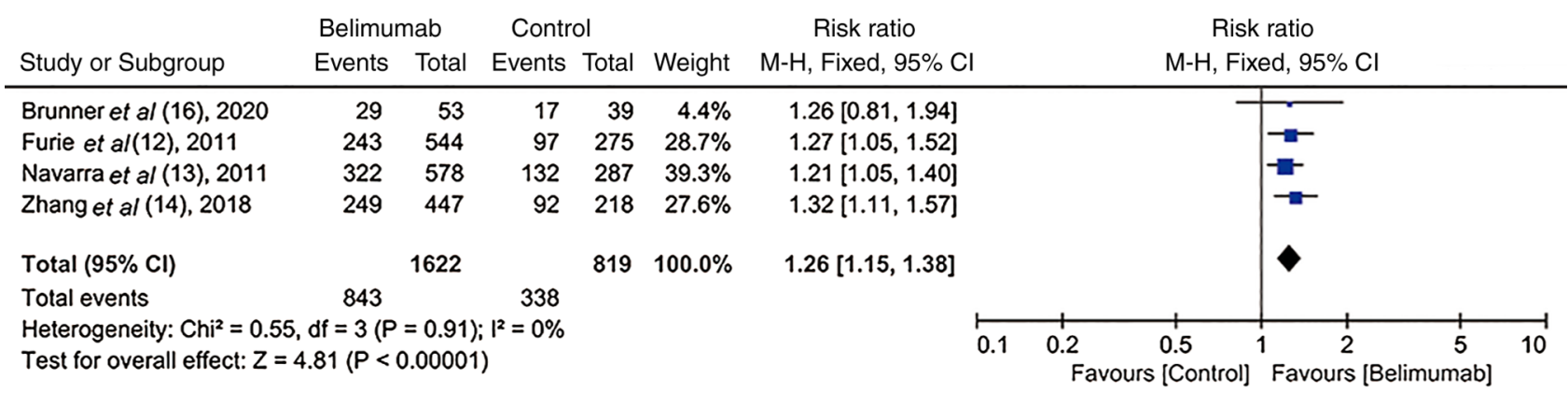

Figure 3. Meta-analysis results of patients with $\geq 4$-point decrease in the Safety of Estrogens in Lupus National Assessment-Systemic Lupus Erythematosus Disease Activity Index score between the belimumab and control groups.

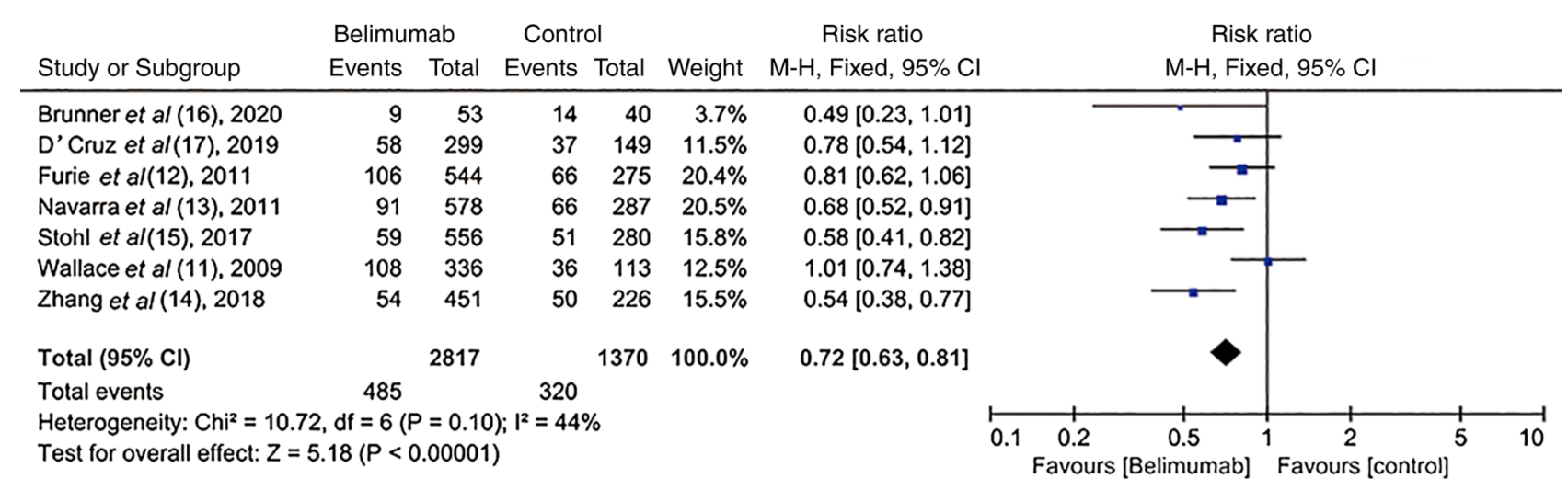

Figure 4. Meta-analysis results of severe flares between the belimumab and control groups.

Meta-analysis of the case series studies. The 19 case series included 2,597 patients with active SLE. A total of 14 of the 19 case series studies involved disease response and the total remission (TR) rate was $60.5 \%$ (95\% CI, 52.1-68.3\%; $\mathrm{P}=0.015$; Fig. 8). Among all the case series studies, $11(19,21,23,24,2$ $6,27,29,32,33,35,36)$ reported changes in the dose of corticosteroids. Therefore, the results revealed that following the treatment of patients with BLM, the use of corticosteroids was significantly decreased (mean difference, $-8.73 ; 95 \% \mathrm{CI},-11.07$ to -6.39 ; $\mathrm{P}<0.00001 ;$ Fig. 9).

\section{Discussion}

SLE is a complex disease and its pathogenesis remains poorly understood. However, it is widely accepted that the activation of autoreactive B- and T-cells, particularly that of B-cells, which may lead to a loss of immune tolerance, plays a crucial role in the pathogenesis of SLE. During the progression of the disease, the activation of cells can promote their proliferation and differentiation into pathogenic cells that produce pathogenic autoantibodies. Therefore, targeting B-cells and their 


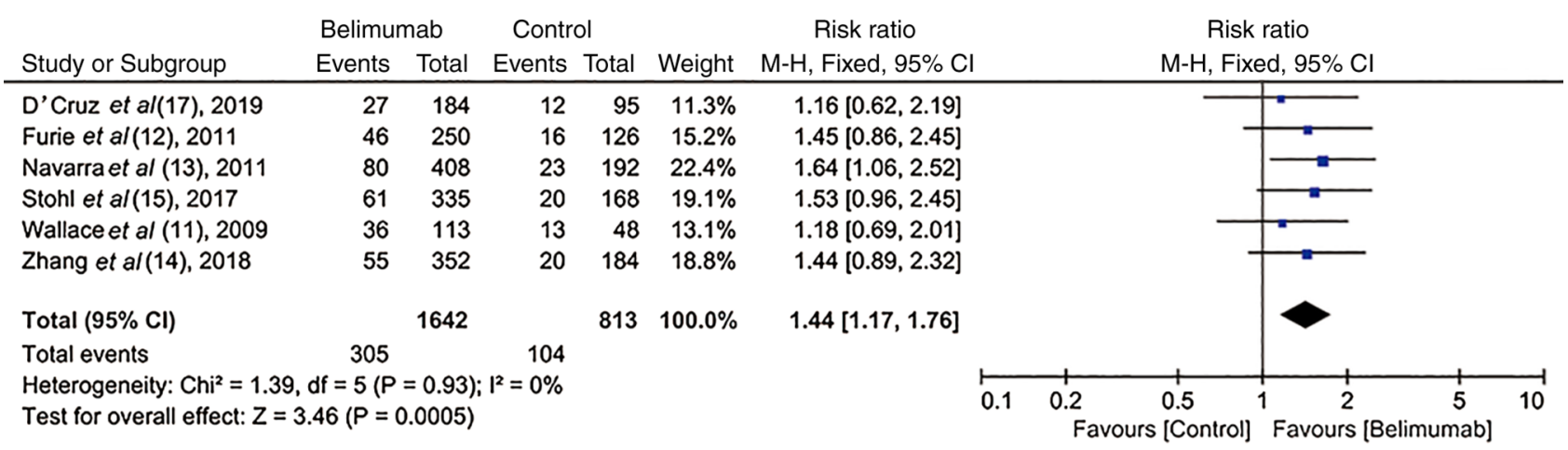

Figure 5. Forest plot illustrating patients with reduced average corticosteroid dosage by $\geq 25$ or 50 to $\leq 7.5 \mathrm{mg} /$ day during weeks $40-52$ between the belimumab and control groups.

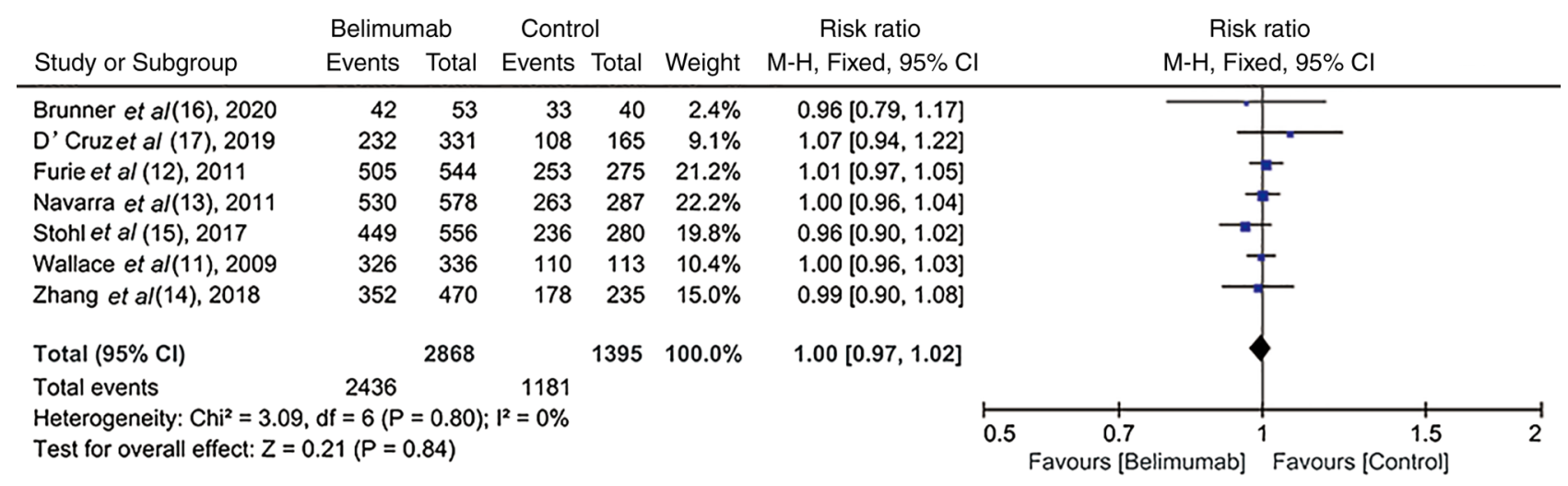

Figure 6. Forest plot illustrating the incidence of adverse events between the belimumab and control groups.

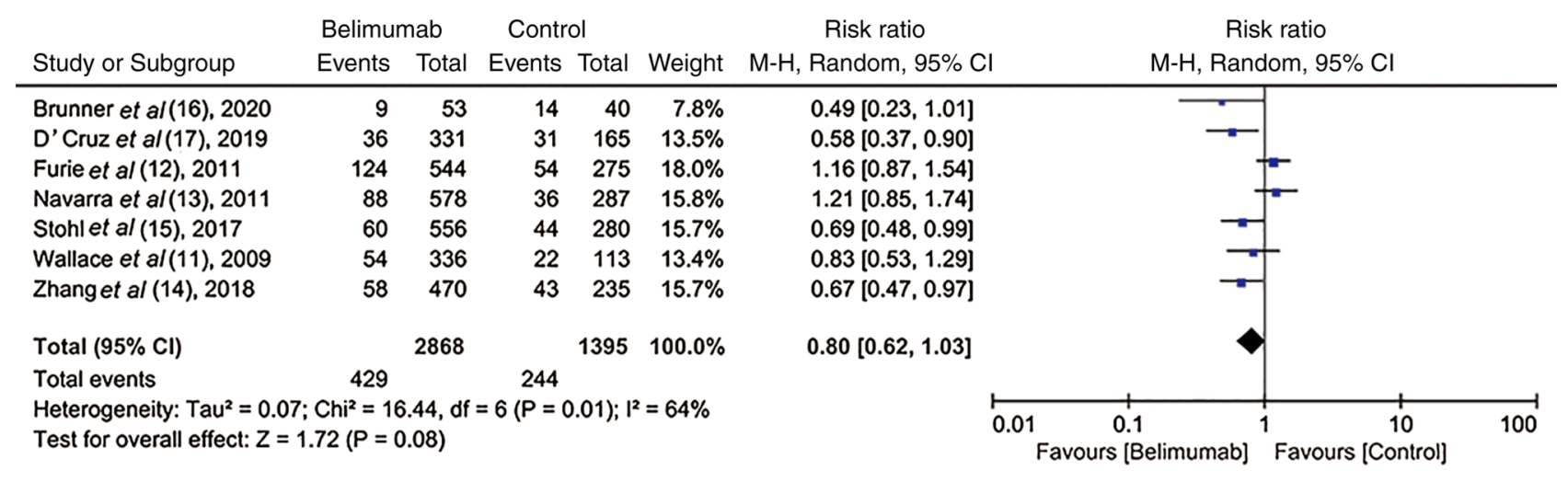

Figure 7. Forest plot illustrating the incidence of serious adverse events between the belimumab and control groups.

related cytokines may be considered as a significant treatment approach. BLyS, a B cell survival factor, plays an essential role in the development of autoimmune SLE by promoting B cell survival, differentiation and maturation (40). Therefore, compared with healthy individuals, patients with SLE exhibit higher BLyS serum concentrations. It has been reported that the plasma levels of BLyS are positively associated with SLE activity (41). BLyS binds to three particular BAFF receptors, namely the transmembrane activator, cyclophilin ligand interactor and B-cell maturation antigen. BLM is a fully humanized monoclonal antibody that specifically binds to soluble trimeric BAFF, thus preventing its interaction with its corresponding receptors, eventually causing autoimmune B-cell apoptosis and reducing new or existing autoimmune B cell clones $(4,5)$.

In the present meta-analysis, seven selected RCTs and 19 case series studies were selected to evaluate the efficacy and safety of BLM plus SOC in patients with active SLE. In the included RCTs, BLM increased the SRI-4 response rate. Furthermore, the SELENA-SLEDAI score, the incidence of severe flare and corticosteroid dosage were significantly decreased in patients with active SLE treated with BLM. Both adults and children treated with intravenous or subcutaneous BLM in combination with SOC exhibited a significant improvement compared with those who received placebo treatment. 


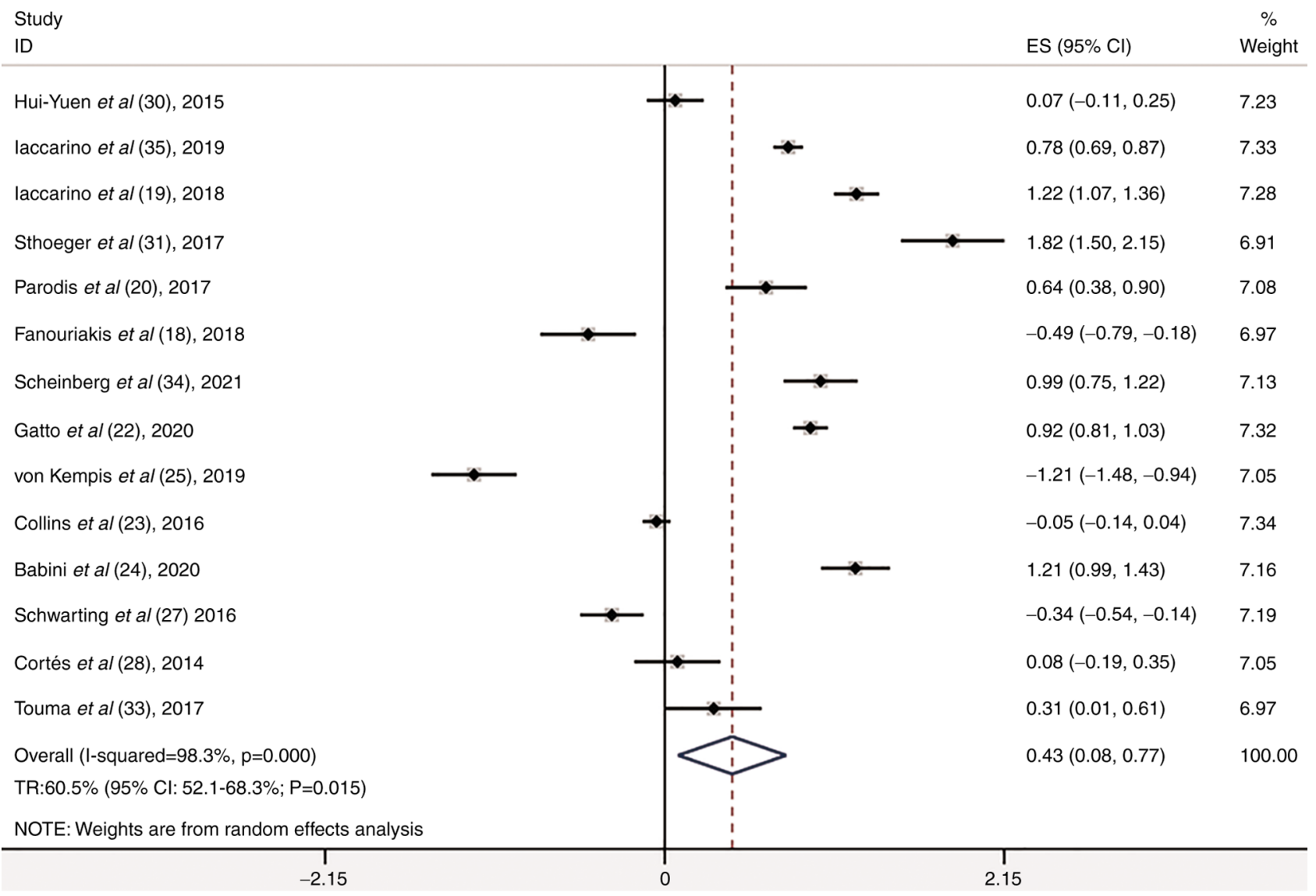

Figure 8. Total remission rate in patients treated with belimumab in the case series studies.

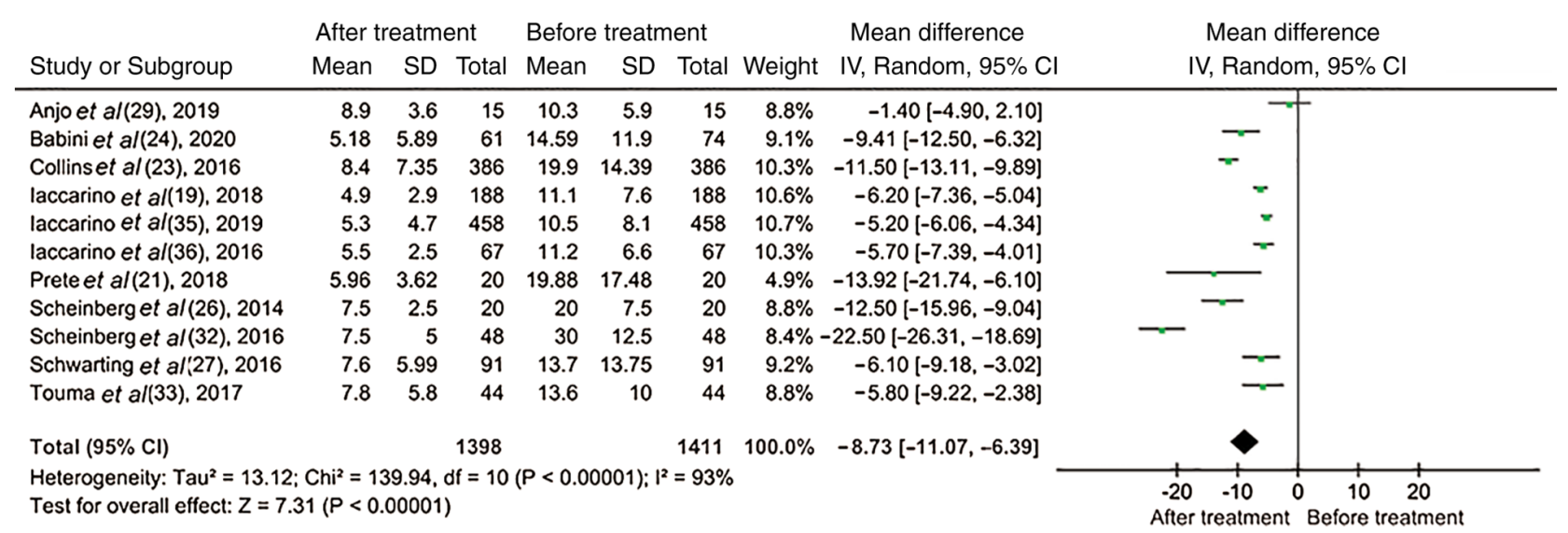

Figure 9. Changes in the dose of corticosteroids in patients treated with belimumab in the case series studies.

Furthermore, BLM combined with SOC was well tolerated from patients with SLE, while no significant differences were observed in the occurrence of AEs and SAEs between the BLM and placebo groups. In the case series studies, the TR was $60.5 \%$ and the use of corticosteroids was significantly reduced following BLM treatment.

SRI is a composite method for evaluating the biological treatment of SLE.This method combines the SELENA-SLEDAI, British Isles Lupus Assessment Group (BILAG) and Physician Global Assessment (PGA) scores, thus offering a more comprehensive assessment of SLE (33). The SRI-4 is defined by a $\geq 4$-point reduction in SELENA-SLEDAI score, no new
BILAG A organ domain score, no $>1$ new BILAG B score and no worsening (increase $<0.3$ ) in PGA score vs. baseline. In the present meta-analysis, the included studies demonstrated that compared with the control group, treatment with BLM notably increased the SRI-4 response rate. Due to the relative strict standards of SRI-4, studies can underestimate the effectiveness of BLM in 'real' world. The OBSErve study, focusing on real-world effectiveness of BLM, verified that BLM could improve the clinical manifestations of SLE (42), thus further supporting the efficacy of BLM in the treatment of SLE.

Currently, glucocorticoids remain the cornerstone of treatment in SLE, particularly when several organs are affected. 
However, emerging evidence has suggested that high doses and the high intensity of glucocorticoid use in patients with SLE can increase the risk of bacterial infection along with other non-infectious complications, such as osteoporosis, sleep disorders and cushingoid syndrome (43). Therefore, the long-term administration of corticosteroids can be burdensome for patients, resulting in a low rate of patient satisfaction and poor compliance (43). Minimizing or even terminating glucocorticoid administration during the treatment of SLE is considered a major goal for scientists. In the present meta-analysis, although none of the six RCTs revealed a statistically significant reduction in glucocorticoid administration, the meta-analysis of the included RCTs revealed that BLM significantly reduced the dose of glucocorticoids administered. Consistent with the RCT analysis, the case series analysis also revealed the same results. In 'real-world' studies, OBSErve demonstrated that the majority of patients could reduce or discontinue oral glucocorticoid use 6 months following BLM treatment. Overall, the aforementioned findings indicated that treatment with BLM effectively reduced glucocorticoid administration in patients with SLE, thus attenuating glucocorticoid-related morbidity and the irreversible damage caused by their use.

The incidence of AEs and SAEs was similar between the BLM and placebo groups. The majority of AEs included infections and infestations. The most common infections in the BLM groups were upper respiratory tract infections, cellulitis, pneumonia and urinary tract infections. As regards the reaction rate at the infusion site, the majority of studies reported that these were the same between both groups. However, a previous study demonstrated that hypersensitivity in the site of infusion was more common in the BLM group compared with the placebo group (14-16 vs. 10\%) (12). Nevertheless, all infusion and hypersensitivity reactions were improved following treatment with antihistamine, prednisone or epinephrine. Psychological effects should be also taken into consideration. In a previous study, a patient who was treated with $1 \mathrm{mg} / \mathrm{kg}$ BLM committed suicide, although this event was not associated with the drug itself (11). In addition, three patients in the BLM group had suicidal intentions, but no one attempted suicide. Additionally, 4 patients in the placebo groups also had suicidal intentions or behavior. Depression was recorded more frequently in patients treated with BLM compared with the placebo group (6-7 vs. 4\%) (12). Furthermore, the incidence of malignant disease was numerically higher in the BLM group compared with the placebo group (9 vs. 2 patients) $(11,12,14)$. However, whether there was an association between BLM and cancer should be further investigated. An extensive study lasting 8 years (BEL112234), also reported a stable safety profile without new safety signals (44). The aforementioned results suggested that BLM was generally well tolerated.

cSLE is less common than adult SLE. However, cSLE is characterized by an enhanced disease activity and immediate neurological, renal and hematological damage $(45,46)$. The trial by Brunner et al (16) revealed that the benefits and risk profile of BLM treatment in children was similar and consistent with those observed in adult patients. Therefore, BLM could be considered as a novel therapeutic approach for treating cSLE. In patients with SLE, disease activity can persist even after the initiation of dialysis. However, the effect of BLM on treating SLE after dialysis has not been previously investigated, at least to the best of our knowledge. The case report study by Karasawa et al (47) demonstrated that SLE activity was attenuated in a patient with SLE treated with BLM following hemodialysis. Another case study on a patient with SLE who was treated with BLM during peritoneal dialysis revealed that the clinical symptoms were significantly improved (48). However, the safety of BLM treatment during dialysis needs to be further evaluated.

The RCTs included four interventions, one study compared treatment with 1,4 and $10 \mathrm{mg} / \mathrm{kg}$ BLM vs. the placebo; two studies, 1 and $10 \mathrm{mg} / \mathrm{kg}$ BLM vs. the placebo; three studies, $10 \mathrm{mg} / \mathrm{kg}$ BLM vs. the placebo; and one study, $200 \mathrm{mg}$ BLM subcutaneous vs. the placebo. The network meta-analysis suggested that the administration of $10 \mathrm{mg} / \mathrm{kg}$ BLM exhibited the highest efficacy in the treatment of active SLE, followed by $1 \mathrm{mg} / \mathrm{kg}$ BLM, $200 \mathrm{mg}$ subcutaneous BLM and placebo (8). Although BLM has emerged as a promising regimen for the treatment of patients with active SLE, in the present meta-analysis, 52.8 and $60.5 \%$ of all patients reached the primary endpoint in the RCTs and case series, respectively. The pathogenesis of SLE is complex and BLM functions by specifically targeting a BAFF. CD20 ${ }^{+}$cells also play a crucial role in the pathogenesis of SLE. Currently, the BEAT Lupus study (trial registration no. ISRCTN47873) aims to investigate the safety and efficacy of BLM in patients treated with rituximab, a B-cell depletion therapy (49). This strategy could provide a novel approach for the treatment of SLE. On the other hand, the follow-up time in the present meta-analysis was relatively short, 52-76 weeks for the RCTs and 6-36 months for the case series. To date, the longest in duration study evaluating the safety and efficacy of BLM was conducted by Wallace et al (50) over the course of 13 years as part of a phase II trial. That study revealed that the percentage of patients who achieved a SRI response, increased from $32.8 \%$ at year 1 to $75.6 \%$ in those who remained under treatment for 12 years. In addition, BLM was well tolerated with no new safety concerns.

Compared with a previous meta-analysis (7), the present study was more comprehensive, including seven RCTs and 19 case series. In addition to the research of RCTs, case series studies based on the 'real' world have more reference significance. A notable finding of the present study was that the use of BLM reduced the use of glucocorticoids. This discovery was not mentioned in the aforementioned previous meta-analysis. Although the present study was an integrated meta-analysis of the clinical research, there were several potential shortcomings. Firstly, the number of patients included in several studies was small and the observation time varied among these studies, potentially leading to a certain degree of uncertainty in the estimation of the TR. Secondly, the possibility of selection and information bias, and uncertain confounders could not be entirely ruled out. Thirdly, in the present meta-analysis, the longest follow-up time was 76 weeks, which was too short to evaluate long-term efficacy. Therefore, further studies with a larger sample size and higher quality, including patients of various ethnicities, undergoing dialysis or during pregnancy need to be performed in the future to further clarify the efficacy and safety of BLM.

In conclusion, the present meta-analysis revealed that BLM therapy may provide significant clinical efficacy and was well tolerated by patients with active SLE. Additionally, treatment with BLM could reduce the use of glucocorticoids. 


\section{Acknowledgements}

Not applicable.

\section{Funding}

No funding was received.

\section{Availability of data and materials}

The datasets used and/or analyzed during the current study are available from the corresponding author on reasonable request.

\section{Authors' contributions}

JZ contributed to the conception, design and modification of the study. JZ, XL and YuX reviewed the articles, extracted the data and organized the database search. XL performed the statistical analysis. JZ wrote the first draft of the manuscript. YaX guided and assisted in the statistical analysis. YaX and HL also contributed to the conception and design of the study. YaX, HL and YuX confirm the authenticity of all the data. All authors contributed to manuscript revision, read, and approved the submitted version.

\section{Ethics approval and consent to participate}

Not applicable.

\section{Patient consent for publication}

Not applicable.

\section{Competing interests}

The authors declare that they have no competing interests.

\section{References}

1. Mok C, Hamijoyo L, Kasitanon N, Chen DY, Chen S, Yamaoka K, Oku K, Li MT, Zamora L, Bae SC, et al: The Asia-pacific league of associations for rheumatology consensus statements on the management of systemic lupus erythematosus. Lancet Rheumatol 3: e517-e531, 2021.

2. Cheema GS, Roschke V, Hilbert DM and Stohl W: Elevated serum B lymphocyte stimulator levels in patients with systemic immune-based rheumatic diseases. Arthritis Rheum 44: 1313-1319, 2001

3. Groom J, Kalled SL, Cutler AH, Olson C, Woodcock SA, Schneider P, Tschopp J, Cachero TG, Batten M, Wheway J, et al Association of BAFF/BLyS overexpression and altered B cell differentiation with Sjögren's syndrome. J Clin Invest 109: 59-68, 2002.

4. Mosak $\mathrm{J}$ and Furie R: Breaking the ice in systemic lupus erythematosus: Belimumab, a promising new therapy. Lupus 22 : 361-371, 2013.

5. Nardelli B, Moore PA, Li Y and Hilbert DM: B lymphocyte stimulator (BLyS): A therapeutic trichotomy for the treatment of B lymphocyte diseases. Leuk Lymphoma 43: 1367-1373, 2002.

6. Vilas-Boas A, Morais SA and Isenberg DA: Belimumab in systemic lupus erythematosus. RMD Open 1: e000011, 2015.

7. Borba HHL, Wiens A, Correr CJ and Pontarolo R: Efficacy and safety of belimumab for the treatment of systemic lupus erythematosus. Value Health 16: PA725-PA726, 2013.
8. Lee YH and Song GG: Comparative efficacy and safety of intravenous or subcutaneous belimumab in combination with standard therapy in patients with active systemic lupus erythematosus: A Bayesian network meta-analysis of randomized controlled trials. Lupus 27: 112-119, 2018.

9. Kandala NB, Connock M, Grove A, Sutcliffe P, Mohiuddin S, Hartley L, Court R, Cummins E, Gordon C and Clarke A: Belimumab: A technological advance for systemic lupus erythematosus patients? Report of a systematic review and meta-analysis. BMJ Open 3: e002852, 2013.

10. Hochberg MC: Updating the American college of rheumatology revised criteria for the classification of systemic lupus erythematosus. Arthritis Rheum 40: 1725, 1997.

11. Wallace DJ, Stohl W, Furie RA, Lisse JR, McKay JD, Merrill JT, Petri MA, Ginzler EM, Chatham WW, McCune WJ, et al: A phase II, randomized, double-blind, placebo-controlled, dose-ranging study of belimumab in patients with active systemic lupus erythematosus. Arthritis Rheum 61: 1168-1178, 2009.

12. Furie R, Petri M, Zamani O, Cervera R, Wallace DJ, Tegzová D, Sanchez-Guerrero J, Schwarting A, Merrill JT, Chatham WW, et al: A phase III, randomized, placebo-controlled study of belimumab, a monoclonal antibody that inhibits B lymphocyte stimulator, in patients with systemic lupus erythematosus. Arthritis Rheum 63: 3918-3930, 2011.

13. Navarra SV, Guzmán RM, Gallacher AE, Hall S, Levy RA, Jimenez RE, Li EK, Thomas M, Kim HY, León MG, et al: Efficacy and safety of belimumab in patients with active systemic lupus erythematosus: A randomised, placebo-controlled, phase 3 trial. Lancet 377: 721-731, 2011.

14. Zhang F, Bae SC, Bass D, Chu M, Egginton S, Gordon D, Roth DA, Zheng J and Tanaka Y: A pivotal phase III, randomised, placebo-controlled study of belimumab in patients with systemic lupus erythematosus located in China, Japan and South Korea. Ann Rheum Dis 77: 355-363, 2018.

15. Stohl W, Schwarting A, Okada M, Scheinberg M, Doria A, Hammer AE, Kleoudis C, Groark J, Bass D, Fox NL, et al: Efficacy and safety of subcutaneous belimumab in systemic lupus erythematosus: A fifty-two-week randomized, double-blind, placebo-controlled study. Arthritis Rheumatol 69: 1016-1027, 2017.

16. Brunner HI, Abud-Mendoza C, Viola DO, Calvo Penades I, Levy D, Anton J, Calderon JE, Chasnyk VG, Ferrandiz MA, Keltsev V, et al: Safety and efficacy of intravenous belimumab in children with systemic lupus erythematosus: Results from a randomised, placebo-controlled trial. Ann Rheum Dis 79: 1340-1348, 2020.

17. D'Cruz D, Maksimowicz-Mckinnon K, Oates J, Santiago MB, Bass D, Burriss S, Gilbride J, Groark J, Miller M and Ji B: 200 Efficacy and safety of belimumab in patients of black race with systemic lupus erythematosus: Results from the EMBRACE study. Lupus Sci Med 6 (Suppl 1): A1-A227, 2019.

18. Fanouriakis A, Adamichou C, Koutsoviti S, Panopoulos S, Staveri C, Klagou A, Tsalapaki C, Pantazi L, Konsta S, Mavragani CP, et al: Low disease activity-irrespective of serologic status at baseline-associated with reduction of corticosteroid dose and number of flares in patients with systemic lupus erythematosus treated with belimumab: A real-life observational study. Semin Arthritis Rheum 48: 467-474, 2018.

19. Iaccarino L, Andreoli L, Bocci EB, Bortoluzzi A, Ceccarelli F, Conti F, De Angelis R, De Marchi G, De Vita S, Di Matteo A, et al: Clinical predictors of response and discontinuation of belimumab in patients with systemic lupus erythematosus in real life setting. Results of a large, multicentric, nationwide study. J Autoimmun 86: 1-8, 2018.

20. Parodis I, Sjöwall C, Jönsen A, Ramsköld D,Zickert A, Frodlund M, Sohrabian A, Arnaud L, Rönnelid J, Malmström V, et al: Smoking and pre-existing organ damage reduce the efficacy of belimumab in systemic lupus erythematosus. Autoimmun Rev 16: 343-351, 2017.

21. Prete M, Leone P, Frassanito MA, Desantis V, Marasco C, Cicco S, Dammacco F, Vacca A and Racanelli V: Belimumab restores Treg/Th17 balance in patients with refractory systemic lupus erythematosus. Lupus 27: 1926-1935, 2018.

22. Gatto M, Saccon F, Zen M, Regola F, Fredi M, Andreoli L, Tincani A, Urban ML, Emmi G, Ceccarelli F, et al: Early disease and low baseline damage as predictors of response to belimumab in patients with systemic lupus erythematosus in a real-life setting. Arthritis Rheumatol 72: 1314-1324, 2020.

23. Collins CE, Dall'Era M, Kan H, Macahilig C, Molta C, Koscielny V and Chang DJ: Response to belimumab among patients with systemic lupus erythematosus in clinical practice settings: 24-Month results from the OBSErve study in the USA. Lupus Sci Med 3: e000118, 2016. 
24. Babini A, Cappuccio AM, Caprarulo C, Casado G Eimon A, Figueredo H, Garcia MA, Magri S, Mannucci P, Perez Rodriguez S, et al: Evaluation of belimumab treatment in patients with systemic lupus erythematosus in a clinical practice setting: Results from a 24-month OBSErve study in Argentina. Lupus 29: 1385-1396, 2020.

25. von Kempis J, Duetsch S, Reuschling N, Villiger R, Villiger PM, Vallelian F, Schaer DJ and Mueller RB: Clinical outcomes in patients with systemic lupus erythematosus treated with belimumab in clinical practice settings: A retrospective analysis of results from the OBSErve study in Switzerland. Swiss Med Wkly 149: w20022, 2019.

26. Scheinberg M and Golmia R: Real life experience on the effect of belimumab in patients with active systemic lupus. Springerplus 3: $758,2014$.

27. Schwarting A, Schroeder JO, Alexander T, Schmalzing M, Fiehn C, Specker C, Perna A, Cholmakow-Bodechtel C, Koscielny VB and Carnarius H: First real-world insights into belimumab use and outcomes in routine clinical care of systemic lupus erythematosus in germany: Results from the OBSErve Germany study. Rheumatol Ther 3: 271-290, 2016.

28. Cortés J, Andreu JL, Calvo J, García-Aparicio AM, Coronell CG and Díaz-Cerezo S: Evaluation of use of belimumab in clinical practice settings (observe study) in spain: Health resource utilization and labour absenteeism. Value Health 17: A534, 2014.

29. Anjo C, Mascaró JM Jr, Espinosa G and Cervera R: Effectiveness and safety of belimumab in patients with systemic lupus erythematosus in a real-world setting. Scand J Rheumatol 48: 469-473, 2019.

30. Hui-Yuen JS, Reddy A, Taylor J, Li X, Eichenfield AH, Bermudez LM, Starr AJ, Imundo LF, Buyon J, Furie RA, et al: Safety and efficacy of belimumab to treat systemic lupus erythematosus in academic clinical practices. J Rheumatol 42 : 2288-2295, 2015.

31. Sthoeger Z, Lorber M, Tal Y, Toubi E, Amital H, Kivity S, Langevitz P, Asher I, Elbirt D and Agmon Levin N: Anti-BLyS treatment of 36 Israeli systemic lupus erythematosus patients. Isr Med Assoc J 19: 44-48, 2017.

32. Scheinberg M, de Melo FF, Bueno AN, Costa CM, de Azevedo Bahr ML and Reis ER: Belimumab for the treatment of corticosteroid-dependent systemic lupus erythematosus: From clinical trials to real-life experience after 1 year of use in 48 Brazilian patients. Clin Rheumatol 35: 1719-1723, 2016.

33. Touma Z, Sayani A, Pineau CA, Fortin I, Matsos M, Ecker GA, Chow A and Iczkovitz S: Belimumab use, clinical outcomes and glucocorticoid reduction in patients with systemic lupus erythematosus receiving belimumab in clinical practice settings: Results from the OBSErve Canada study. Rheumatol Int 37 865-873, 2017.

34. Scheinberg MA, Golmia AP, Golmia RP, de Souza Molotievschi RN and Dos Santos Cortada AP: Lupus low disease activity (SLE) in patients treated with belimumab: A single-center real-life experience (2016-2019). Clin Rheumatol 40: 923-927, 2021

35. Iaccarino L, Saccon F, Mathieu A, Piga M, Ceribelli A, Selmi C, Cardinaletti P, Gabrielli A, Di Matteo A, De Angelis R, et al: FRI0199 effectiveness and safety of belimumab in patientswith active systemic lupus erythematosus: Results from a large, nationwide, multicentric study. Ann Rheum Dis 78 (Suppl 2): S778-S779, 2019.

36. Iaccarino L, Bettio S, Reggia R, Zen M, Frassi M, Andreoli L, Gatto M, Piantoni S, Nalotto L, Franceschini F, et al: Belimumab decreases flare rate and hinders the expected damage progression in patients with active systemic lupus erythematosus. Arthritis Care Res 69: 115-123, 2016.

37. Buyon JP, Petri MA, Kim MY, Kalunian KC, Grossman J, Hahn BH, Merrill JT, Sammaritano L, Lockshin M, Alarcón GS, et al: The effect of combined estrogen and progesterone hormone replacement therapy on disease activity in systemic lupus erythematosus: A randomized trial. Ann Intern Med 142: 953-962, 2005
38. Petri M, Buyon J and Kim M: Classification and definition of major flares in SLE clinical trials. Lupus 8: 685-691, 1999.

39. Petri M, Kim MY, Kalunian KC, Grossman J, Hahn BH, Sammaritano LR, Lockshin M, Merrill JT, Belmont HM, Askanase AD, et al: Combined oral contraceptives in women with systemic lupus erythematosus. N Engl J Med 353: 2550-2558, 2005.

40. Vincent FB, Saulep-Easton D, Figgett WA, Fairfax KA and Mackay F: The BAFF/APRIL system: Emerging functions beyond B cell biology and autoimmunity. Cytokine Growth Factor Rev 24: 203-215, 2013

41. Lee WS and Amengual O: B cells targeting therapy in the management of systemic lupus erythematosus. Immunol Med 43: 16-35, 2020.

42. Collins CE, Cortes-Hernández J, Garcia MA, von Kempis J, Schwarting A, Touma Z, Kurtinecz M and Gairy K: Real-world effectiveness of belimumab in the treatment of systemic lupus erythematosus: Pooled analysis of multi-country data from the OBSErve studies. Rheumatol Ther 7: 949-965, 2020.

43. Chen HL, Shen LJ, Hsu PN, Shen CY, Hall SA and Hsiao FY Cumulative burden of glucocorticoid-related adverse events in patients with systemic lupus erythematosus: Findings from a 12-year longitudinal study. J Rheumatol 45: 83-89, 2018.

44. van Vollenhoven RF, Navarra SV, Levy RA, Thomas M, Heath A, Lustine T, Adamkovic A, Fettiplace J, Wang ML, Ji B and Roth D: Long-term safety and limited organ damage in patients with systemic lupus erythematosus treated with belimumab: A phase III study extension. Rheumatology (Oxford) 59: 281-291, 2020.

45. Kamphuis S and Silverman ED: Prevalence and burden of pediatric-onset systemic lupus erythematosus. Nat Rev Rheumatol 6: 538-546, 2010

46. Silva CA, Avcin T and Brunner HI: Taxonomy for systemic lupus erythematosus with onset before adulthood. Arthritis Care Res (Hoboken) 64: 1787-1793, 2012.

47. Karasawa K, Ogura S, Takabe T, Miyabe Y, Iwabuchi Y, Akiyama K, Sato M, Moriyama T, Uchida K and Nitta K: Successful treatment with belimumab in a patient with refractory systemic lupus erythematosus after initiation of hemodialysis: Considering the synergistic effect of belimumab and immunological burn-out phenomenon in end-stage renal disease patients on hemodialysis. Blood Purif: Apr 23, 2021 (Epub ahead of print). doi: 10.1159/000512585.

48. Binda V, Trezzi B, Del Papa N, Beretta L, Frontini G, Porata G, Fabbrini P, Pozzi MR, Messa P, Sinico RA and Moroni G: Belimumab may decrease flare rate and allow glucocorticoid withdrawal in lupus nephritis (including dialysis and transplanted patient). J Nephrol 33: 1019-1025, 2020.

49. Jones A, Muller P, Dore CJ, Ikeji F, Caverly E, Chowdhury K, Isenberg DA, Gordon C and Ehrenstein MR: Belimumab after $B$ cell depletion therapy in patients with systemic lupus erythematosus (BEAT Lupus) protocol: A prospective multicentre, double-blind, randomised, placebo-controlled, 52-week phase II clinical trial. BMJ Open 9: e032569, 2019.

50. Wallace DJ, Ginzler EM, Merrill JT, Furie RA, Stohl W, Chatham WW, Weinstein A, McKay JD, McCune WJ, Petri M, et al: Safety and efficacy of belimumab plus standard therapy for up to thirteen years in patients with systemic lupus erythematosus. Arthritis Rheumatol 71: 1125-1134, 2019.

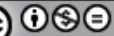

This work is licensed under a Creative Commons Attribution-NonCommercial-NoDerivatives 4.0 International (CC BY-NC-ND 4.0) License. 